\title{
Applications of Fuzzy Sliding Mode Control for a Gyroscope System
}

\author{
Shih-Chung Chen, ${ }^{1}$ Chao-Lin Kuo, ${ }^{2}$ Chia-Hung Lin, ${ }^{3}$ \\ Chih-Hung Hsu, ${ }^{1}$ and Chien-Kuo Tsui ${ }^{2}$ \\ ${ }^{1}$ Department of Electrical Engineering, Southern Taiwan University of Science and Technology, Tainan 71005, Taiwan \\ ${ }^{2}$ Department of Maritime Information and Technology, National Kaohsiung Marine University, Kaohsiung 80543, Taiwan \\ ${ }^{3}$ Department of Electrical Engineering, Kao Yuan University, Kaohsiung 82151, Taiwan
}

Correspondence should be addressed to Chao-Lin Kuo; clkuo@mail.nkmu.edu.tw

Received 13 June 2013; Accepted 29 July 2013

Academic Editor: Chang-Hua Lien

Copyright (c) 2013 Shih-Chung Chen et al. This is an open access article distributed under the Creative Commons Attribution License, which permits unrestricted use, distribution, and reproduction in any medium, provided the original work is properly cited.

The study proposed the application of the fuzzy sliding mode for a gyroscope system status control. The state response analysis of the gyroscope system revealed highly nonlinear and chaotic subharmonic motions of $2 T$ during state formation. The current study discussed the use of tracking control on the sliding mode control and fuzzy sliding mode control of a gyroscope control system. Consequently, the gyroscope system drives from chaotic motion to periodic motion. The numerical simulation results confirm that the proposed controller provides good system stability and convergence without chattering phenomena.

\section{Introduction}

Since most systems in the natural world are nonlinear, presenting them in a linear manner is challenging. Some systems exhibit chaotic phenomena after a certain number of intervals. The chaos phenomenon has been widely discussed and researched after many areas, including mechanical engineering, electrical engineering, and communications. Its main feature is that different initial conditions can produce large differences in the system response. According to [1-3] certain conditions generate chaotic behavior in a gyroscope system, which results in system instability and design problems. Wang and Yau used differential transformation (DT) method and Runge-Kutta (RK) method to analyze the dynamic behavior of the gyroscope system [1]. They also proposed the use of a sliding mode controller to stabilize the system and to change its state from chaotic-driven motion to periodic motion. Ge and Lee used the Lyapunov stability theory and adaptive control and random optimization method to synchronize two identical gyroscope systems and to track system parameters [2]. Fei et al. based the adaptive sliding mode control on a radial basis function neural network for threeaxis gyroscope used in micro electromechanical systems (MEMS) [4]. Chaos control has attracted the attention of many scholars in recent years [5-13]. Yau et al. used particle swam optimization (PSO) algorithm to find close optimal parameters for PID control and used a PID controller for chaos synchronization control [7]. Aghababa et al. proposed the use of sliding mode control technology to solve finite-time chaos synchronization problems by enabling synchronization of two different chaotic systems with unknown parameters [9]. Li and Kumar proposed the application of nonsingular fast terminal sliding mode control technology in satellite attitude synchronization [10].

This study proposes the use of fuzzy sliding mode control technology for state control of a gyroscope system. Formation of chaotic motion in a gyroscope during subharmonic motions of $2 T$ is discussed, and controller designs that drive the gyroscope system state from chaotic motion to periodic motion are proposed. The framework of this paper is as follows: Section 2 briefly introduces the gyroscope system and its issues; Section 3 introduces the 
sliding mode control in the gyroscope system design and its applications; Section 4 proposes the fuzzy sliding mode control for a gyroscope system and introduces the application of the Lyapunov stability theory for designing a fuzzy sliding mode controller; and Section 5 presents the results of the numerical simulation approach used to verify the feasibility of the proposed method and states the conclusions of the study.

\section{A Gyroscope System}

Figure 1 shows the heavy symmetric gyroscope placed on the structure of the vibration mechanism. The Euler angles $\theta, \psi$, and $\varphi$ refer to the three-dimensional motion of the system, and multiple harmonic motion $\sum_{k=1}^{n} A_{k} \sin \omega_{k} t$ describes the base of the vibrations. The status order is $x=\theta, y=\dot{\theta}$, and $z=\dot{\psi}$. The system dynamic equations are as follows [1]:

$$
\begin{gathered}
\dot{x}=y, \\
\dot{y}=-\frac{\left(\beta_{\psi}-\beta_{\varphi} \cos x\right)\left(\beta_{\varphi}-\beta_{\psi} \cos x\right)}{I_{1}^{2} \cdot \sin ^{3} x}-\frac{C}{I_{1}} y \\
+\frac{M_{g} l}{I_{1}} \sin x-\frac{M_{g}}{I_{1}} \sum_{k=1}^{n} A_{k} \sin \left(\omega_{k} t\right) \cdot \sin x, \\
\dot{z}=-\frac{2 \cos x}{\sin x} y z+\frac{\beta_{\varphi} y}{I_{1} \sin x},
\end{gathered}
$$

where $I_{1}$ is the uniform gyroscope moment of inertia axis, $M_{g}$ is the weight of gravity, and $l$ is the distance between the center of the gyroscope and point $O$. If only the system of the subharmonic motions of $2 T$ is considered, the system can be rewritten as

$$
\begin{gathered}
\dot{x}=y, \\
\dot{y}=-\frac{\left(\beta_{\psi}-\beta_{\varphi} \cos x\right)\left(\beta_{\varphi}-\beta_{\psi} \cos x\right)}{I_{1}^{2} \cdot \sin ^{3} x}-\frac{C}{I_{1}} y \\
+\frac{M_{g} l}{I_{1}} \sin x-\frac{M_{g}}{I_{1}} A_{2} \sin \left(\omega_{2} t\right) \sin x, \\
\dot{z}=-\frac{2 \cos x}{\sin x} y z+\frac{\beta_{\varphi} y}{I_{1} \sin x} .
\end{gathered}
$$

The system parameter settings are $\beta_{\psi}=2, \beta_{\varphi}=5, I_{1}=1$, $M_{g}=4, l=0.25, C=0.5$, and $\omega_{2}=2$. The analysis in [1] showed that the chaos phenomenon in a gyroscope system results from subharmonic motions of $2 T$. Based on the dynamic analysis of [1], $A_{2}=14.21$ was selected for the study. Figure 2 shows the numerical simulation results for the phase plane trajectory of the gyroscope [2]. The results indicate that chaos phenomena resulted from two attractors of system trajectories.

Since the gyroscope system has chaotic motion, driving its state into periodic motion requires the addition of $u_{1}(t)$ and $u_{2}(t)$ to the second and third items of the control,

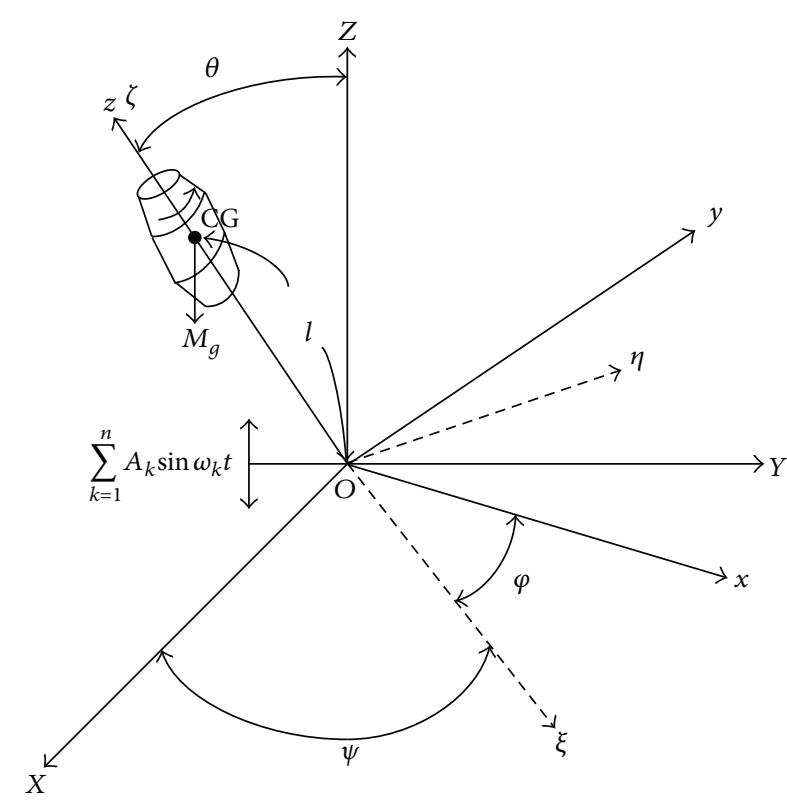

FIGURE 1: A schematic diagram of a heavy symmetric gyroscope [2].

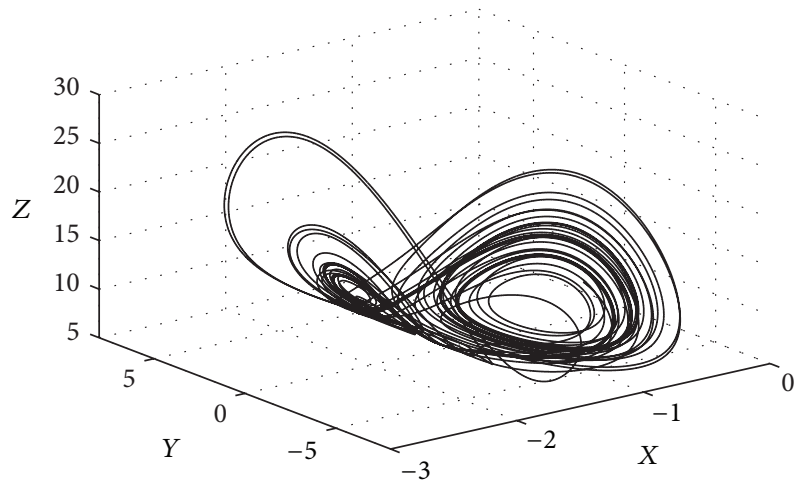

FIGURE 2: Phase portrait of gyroscope system.

respectively. The dynamic equation for the system can then be rewritten as

$$
\begin{gathered}
\dot{x}=y, \\
\dot{y}=-\frac{\left(\beta_{\psi}-\beta_{\varphi} \cos x\right)\left(\beta_{\varphi}-\beta_{\psi} \cos x\right)}{I_{1}^{2} \cdot \sin ^{3} x}-\frac{C}{I_{1}} y \\
+\frac{M_{g} l}{I_{1}} \sin x-\frac{M_{g}}{I_{1}} A_{2} \sin \left(\omega_{2} t\right) \sin x+u_{1}(t), \\
\dot{z}=-\frac{2 \cos x}{\sin x} y z+\frac{\beta_{\varphi} y}{I_{1} \sin x}+u_{2}(t) .
\end{gathered}
$$

A controller was designed into the system to solve two consecutive equations for tracking $\theta_{d}$ and $\psi_{d}$ in the system, which allows the gyroscope system to exhibit periodic motion. Through the system control input and state response, the tracking error can be defined as $E(t)=\left[\begin{array}{lll}e_{1} & e_{2} & e_{3}\end{array}\right]^{T}=$ $\left[\begin{array}{lll}x-\theta_{d} & y-\dot{\theta}_{d} & z-\dot{\psi}_{d}\end{array}\right]^{T}$. By integrating the system dynamic 
equations and system errors equations, the error dynamic equation can be computed as

$$
\begin{aligned}
\dot{e}_{1}=e_{2}, & \\
\dot{e}_{2}=- & \frac{\left(\beta_{\psi}-\beta_{\varphi} \cos \left(e_{1}+\theta_{d}\right)\right)\left(\beta_{\varphi}-\beta_{\psi} \cos \left(e_{1}+\theta_{d}\right)\right)}{I_{1}^{2} \cdot \sin ^{3}\left(e_{1}+\theta_{d}\right)} \\
- & \frac{C}{I_{1}}\left(e_{2}+\dot{\theta}_{d}\right) \\
+ & \frac{M_{g} l}{I_{1}}\left(l-A_{2} \cdot \sin \omega_{2} t\right) \cdot \sin \left(e_{1}+\dot{\theta}_{d}\right) \\
- & \ddot{\theta}_{d}+u_{1}(t), \\
\dot{e}_{3}= & -\frac{2 \cos \left(e_{1}+\theta_{d}\right)}{\sin \left(e_{1}+\theta_{d}\right)} \cdot\left(e_{2}+\dot{\theta}_{d}\right) \cdot\left(e_{3}+\dot{\psi}_{d}\right) \\
& +\frac{\beta_{\varphi}\left(e_{2}+\dot{\theta}_{d}\right)}{I_{1} \sin \left(e_{1}+\theta_{d}\right)}-\ddot{\psi}_{d}+u_{2}(t) .
\end{aligned}
$$

Definition 1. Considering the design of the state signal and the gyroscope system in (3)-(5), if $T=T(E(0))>0$ exists, then

$$
\lim _{t \geq T}\|E(t)\|=0
$$

If $t \geq T$, then the gyroscope state track to the state signal design within a limited time.

This study designed a fuzzy sliding mode controller to enable the output state of the gyroscope system to tracking the design state. Tracking control is achieved by using the controller for gradual convergence of the error dynamics equation to zero.

\section{Sliding Mode Control}

In accordance with the analysis of chaotic phenomena in a gyroscope system, Wang and Yau proposed the use of sliding mode controller to tracking system status and harmonic signals [1]. To ensure system stability and error convergence to zero, two sliding surfaces were defined:

$$
\begin{gathered}
s_{s 1}(t)=e_{2}(t)+\lambda_{1} e_{1}(t), \\
s_{s 2}(t)=e_{3}(t)+\lambda_{2} \int_{0}^{t} e_{1}(\tau) d \tau,
\end{gathered}
$$

where $\lambda_{1}$ and $\lambda_{2}$ are positive constants. When the system acts on the sliding surface, (8) are satisfied as

$$
\begin{gathered}
s_{s 1}(t)=e_{2}(t)+\lambda_{1} e_{1}(t)=0 \\
s_{s 2}(t)=e_{3}(t)+\lambda_{2} \int_{0}^{t} e_{1}(\tau) d \tau=0 .
\end{gathered}
$$

Equations (9) can calculate the equivalent control for the system $u_{\text {sleq }}(t)$ and $u_{s 2 \mathrm{eq}}(t)$. The equivalent control can drive the system dynamic error to maintain the gradual movement of the sliding surface toward the origin. It can be deducted that this ensures that the dynamic system error progressively approaches zero. In order to make the system error status contact the designed sliding surface, the system selects two sliding mode control inputs, which is designed as

$$
\begin{array}{ll}
u_{s 1}=-\gamma_{1} \alpha_{1} \operatorname{sgn}\left(s_{s 1}(t)\right), & \gamma_{1}>0, \\
u_{s 2}=-\gamma_{2} \alpha_{2} \operatorname{sgn}\left(s_{s 2}(t)\right), & \gamma_{2}>0 .
\end{array}
$$

Based on [1], $\alpha_{1}$ and $\alpha_{2}$ are

$$
\begin{aligned}
\alpha_{1}= & \mid \frac{\left(\beta_{\psi}-\beta_{\varphi} \cos \left(e_{1}+\theta_{d}\right)\right)\left(\beta_{\varphi}-\beta_{\psi} \cos \left(e_{1}+\theta_{d}\right)\right)}{I_{1}^{2} \cdot \sin ^{3}\left(e_{1}+\theta_{d}\right)} f \\
& +\left(\frac{C}{I_{1}}-\lambda_{1}\right) e_{2}\left|+\frac{M_{g}}{I_{1}}\left(l+A_{2}\right)+\right| \frac{C}{I_{1}} \dot{\theta}_{d}|+| \ddot{\theta}_{d} \mid, \\
\alpha_{2}=\mid- & {\left[\frac{2 \cos \left(e_{1}+\theta_{d}\right)}{\sin \left(e_{1}+\theta_{d}\right)}\left(e_{2}+\theta_{d}\right)\left(e_{3}+\dot{\psi}_{d}\right)\right] } \\
& +\lambda_{2} e_{3}+\frac{\beta_{\varphi}\left(e_{2}+\dot{\theta}_{d}\right)}{I_{1} \sin \left(e_{1}+\theta_{d}\right)}|+| \ddot{\psi}_{d} \mid .
\end{aligned}
$$

To prevent the $\operatorname{sgn}(\bullet)$ equation from forming a noncontinuous control signal in the design, which would cause chattering phenomenon in the system, the controller presented in (10) can be modified as

$$
\begin{array}{ll}
u_{s 1}=-\gamma_{1} \alpha_{1} \frac{s_{s 1}}{\left|s_{s 1}\right|+\delta_{1}}, & \gamma_{1}>1, \\
u_{s 2}=-\gamma_{2} \alpha_{2} \frac{s_{s 2}}{\left|s_{s 2}\right|+\delta_{2}}, & \gamma_{2}>1 .
\end{array}
$$

$\delta_{1}$ and $\delta_{2}$ are small constants and are designed to prevent the denominator from becoming zero.

\section{Fuzzy Sliding Mode Control}

This section describes the design and implementation of a fuzzy sliding mode controller in the gyroscope system. First, the two controller inputs for the gyroscope were designed for the two sliding planes as follows:

$$
\begin{aligned}
& s_{f 1}(t)=e_{2}(t)+\int_{0}^{t}\left(e_{2}(\tau)+k_{1} e_{1}(\tau)\right) d \tau, \\
& s_{f 2}(t)=e_{3}(t)+\int_{0}^{t}\left(k_{2} e_{3}(\tau)+e_{1}(\tau)\right) d \tau,
\end{aligned}
$$

where $k_{i} \in R, i=1,2$ are positive numbers. Let (13) and (14) be equal to zero and they can easily fine equivalent control as

$$
\begin{aligned}
u_{\mathrm{feq} 1}= & \frac{\left(\beta_{\psi}-\beta_{\varphi} \cos \left(e_{1}+\theta_{d}\right)\right)\left(\beta_{\varphi}-\beta_{\psi} \cos \left(e_{1}+\theta_{d}\right)\right)}{I_{1}^{2} \cdot \sin ^{3}\left(e_{1}+\theta_{d}\right)} \\
& +\frac{C}{I_{1}}\left(e_{2}+\dot{\theta}_{d}\right)-\frac{M_{g}}{I_{1}}\left(l-A_{2} \sin \omega t\right) \\
& \cdot \sin \left(e_{1}+\theta_{d}\right)+\ddot{\theta}_{d}-e_{2}-k_{1} e_{1},
\end{aligned}
$$




$$
\begin{aligned}
u_{\mathrm{feq} 2}= & \frac{2 \cos \left(e_{1}+\theta_{d}\right)}{\sin \left(e_{1}+\theta_{d}\right)}\left(e_{2}+\dot{\theta}_{d}\right)\left(e_{3}+\dot{\psi}_{d}\right) \\
& -\frac{\beta_{\varphi}\left(e_{2}+\dot{\theta}_{d}\right)}{I_{1} \cdot \sin \left(e_{1}+\theta_{d}\right)}+\ddot{\psi}_{d}-k_{2} e_{3}-e_{1} .
\end{aligned}
$$

When the gyroscope system error states are during the sliding surface design, the controllers in (16) can be used to maintain the system states in sliding surfaces. Two fuzzy sliding mode controllers are used so that the error state quickly comes into contact with the sliding surfaces:

$$
\begin{gathered}
u_{f 1}=\exp \left(\frac{e_{2}-e_{1}}{\varepsilon_{1}}\right) \operatorname{Fuz}\left(\frac{S_{f 1}}{\varepsilon_{s 1}}\right), \\
u_{f 2}=\exp \left(\frac{e_{3}}{\varepsilon_{2}}\right) \operatorname{Fuz}\left(\frac{S_{f 2}}{\varepsilon_{s 2}}\right),
\end{gathered}
$$

where $\varepsilon_{i} \in R, i=1,2$ are strictly positive constants. The $\varepsilon_{s i}$ is a normalization factor greater than zero. The $\operatorname{Fuz}(\bullet)$ is a fuzzy controller. A fuzzy controller was designed using the following 7 IF-THEN control rules:

$$
\begin{aligned}
& \mathrm{R}_{1} \text { : if } \frac{S_{f i}}{\varepsilon_{s i}} \text { is } \mathrm{NB} \text {, then } \operatorname{Fuz}(\bullet) \text { is } \mathrm{PB} \text {; } \\
& \mathrm{R}_{2} \text { : if } \frac{S_{f i}}{\varepsilon_{s i}} \text { is } \mathrm{NM} \text {, then } \operatorname{Fuz}(\bullet) \text { is } \mathrm{PM} \text {; } \\
& \mathrm{R}_{3} \text { : if } \frac{S_{f i}}{\varepsilon_{s i}} \text { is NS, then } \operatorname{Fuz}(\bullet) \text { is PS; } \\
& \mathrm{R}_{4} \text { : if } \frac{S_{f i}}{\varepsilon_{s i}} \text { is } \mathrm{ZE} \text {, then } \mathrm{Fuz}(\bullet) \text { is } \mathrm{ZE} \text {; } \\
& \mathrm{R}_{5} \text { : if } \frac{S_{f i}}{\varepsilon_{s i}} \text { is PS, then } \operatorname{Fuz}(\bullet) \text { is NS; } \\
& \mathrm{R}_{6} \text { : if } \frac{S_{f i}}{\varepsilon_{s i}} \text { is } \mathrm{PM} \text {, then } \operatorname{Fuz}(\bullet) \text { is } \mathrm{NM} \text {, } \\
& \mathrm{R}_{7} \text { : if } \frac{S_{f i}}{\varepsilon_{s i}} \text { is } \mathrm{PB} \text {, then } \operatorname{Fuz}(\bullet) \text { is } \mathrm{NB} \text {, }
\end{aligned}
$$

where NB, NM, NS, ZE, PS, PM, and PB are colloquial narratives representing negative big, negative medium, negative small, zero, positive small, positive medium, and positive big, respectively. The $S_{f i} / \varepsilon_{s i}$ is a triangle-shaped fuzzy number, controller inference corresponds to fuzzy singleton, and the weighted average defuzzification method is used to calculate the controller output. Through addition of fuzzy controller design to control input, $u_{1}(t)$ and $u_{2}(t)$ of the gyroscope system (4) and (5) can be designed as

$$
\begin{aligned}
& u_{1}(t)=u_{\text {feq } 1}(t)+u_{f 1}(t), \\
& u_{2}(t)=u_{\text {feq } 2}(t)+u_{f 2}(t) .
\end{aligned}
$$

Adjusting the fuzzy controller can drive the gyroscope system state to tracking the design state.
Theorem 2. Considering the error dynamics equations (6) for the gyroscope system and the designed control inputs (19), the gyroscope system asymptotically synchronizes with the designed signal so that the system error converges to zero.

Proof. A Lyapunov function is defined as

$$
V(t)=\frac{S_{f 1}^{2}}{2}+\frac{S_{f 2}^{2}}{2} .
$$

Differentiating (20) with respect to time yields

$$
\begin{aligned}
& \dot{V}=S_{f 1} \dot{S}_{f 1}+S_{f 2} \dot{S}_{f 2} \\
& =S_{f 1}\left(\dot{e}_{2}(t)+e_{2}(t)+k_{1} e_{1}(t)\right) \\
& +S_{f 2}\left(\dot{e}_{3}(t)+k_{2} e_{3}(t)+e_{1}(t)\right) \\
& =S_{f 1}\left(-\frac{\left(\beta_{\psi}-\beta_{\varphi} \cos \left(e_{1}+\theta_{d}\right)\right)\left(\beta_{\varphi}-\beta_{\psi} \cos \left(e_{1}+\theta_{d}\right)\right)}{I_{1}^{2} \cdot \sin ^{3}\left(e_{1}+\theta_{d}\right)}\right. \\
& -\frac{C}{I_{1}}\left(e_{2}+\dot{\theta}_{d}\right) \\
& +\frac{M_{g} l}{I_{1}}\left(l-A_{2} \cdot \sin \omega_{2} t\right) \cdot \sin \left(e_{1}+\dot{\theta}_{d}\right) \\
& \left.-\ddot{\theta}_{d}+u_{1}+e_{2}+k_{1} e_{1}\right) \\
& +S_{f 2}\left(-\frac{2 \cos \left(e_{1}+\theta_{d}\right)}{\sin \left(e_{1}+\theta_{d}\right)} \cdot\left(e_{2}+\dot{\theta}_{d}\right) \cdot\left(e_{3}+\dot{\psi}_{d}\right)\right. \\
& +\frac{\beta_{\varphi}\left(e_{2}+\dot{\theta}_{d}\right)}{I_{1} \sin \left(e_{1}+\theta_{d}\right)} \\
& \left.-\ddot{\psi}_{d}+u_{2}(t)+k_{2} e_{3}(t)+e_{1}(t)\right) \text {. }
\end{aligned}
$$

Substituting (19) obtains

$$
\begin{aligned}
\dot{V}= & S_{f 1} \exp \left(\frac{e_{2}-e_{1}}{\varepsilon_{1}}\right) \operatorname{Fuz}\left(\frac{S_{f 1}}{\varepsilon_{s 1}}\right) \\
& +S_{f 2} \exp \left(\frac{e_{3}}{\varepsilon_{2}}\right) \operatorname{Fuz}\left(\frac{S_{f 2}}{\varepsilon_{s 2}}\right) \\
\leq & -\exp \left(\frac{e_{2}-e_{1}}{\varepsilon_{1}}\right)\left|S_{f 1}\right|-\exp \left(\frac{e_{3}}{\varepsilon_{2}}\right)\left|S_{f 2}\right| \\
& <0 .
\end{aligned}
$$

Since $\exp \left(\left(e_{2}-e_{1}\right) / \varepsilon_{1}\right)>0$ and $\exp \left(e_{3} / \varepsilon_{2}\right)>0$, its guarantee $\dot{V}<0$. The states errors of the system are ensured to converge to zero. This completes the proof.

To verify the performance merits of the designed fuzzy sliding controller, integral absolute error (IAE) was used as 


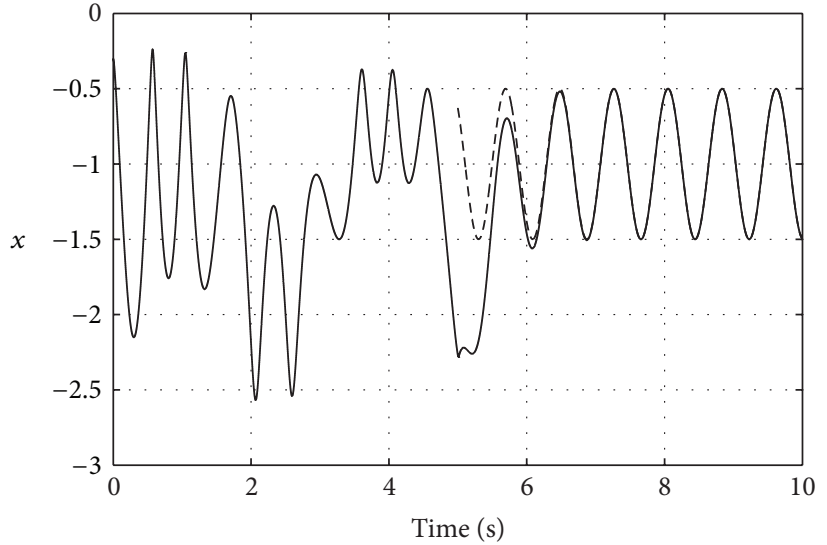

(a)

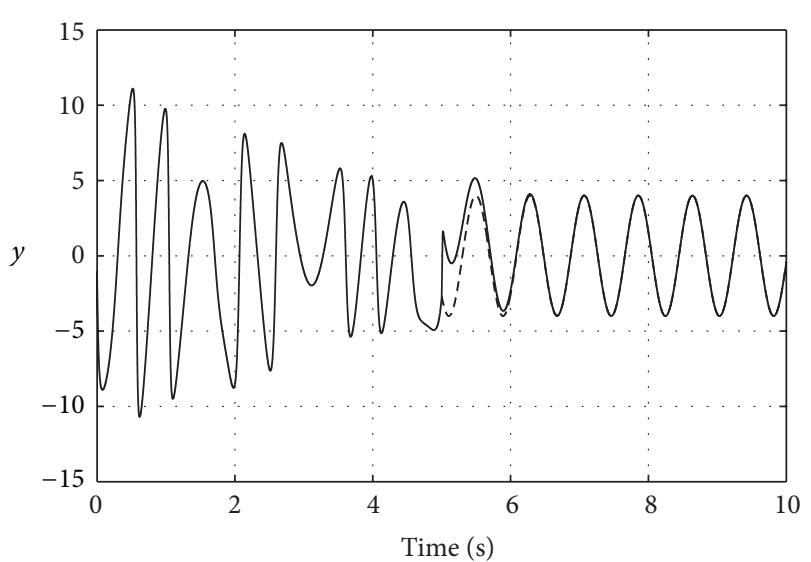

(b)

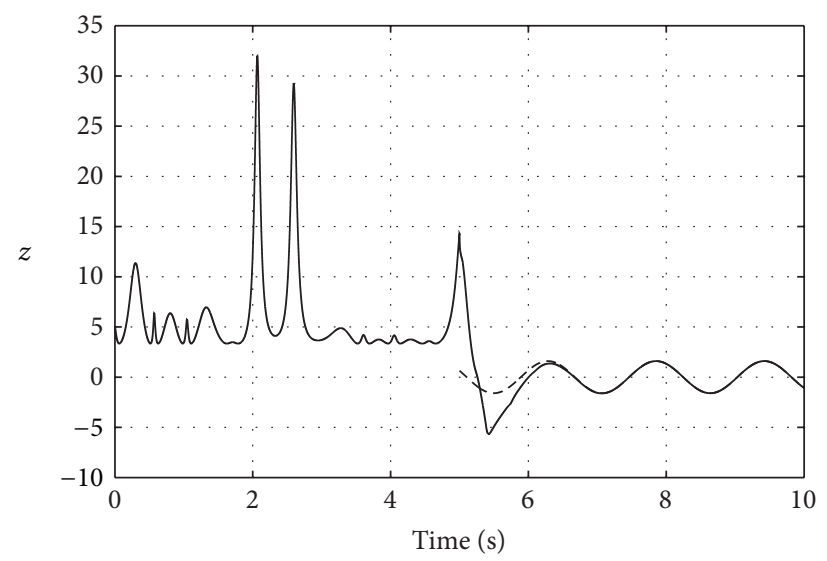

(c)

FIGURE 3: Time response of gyroscope via sliding mode control.

a performance index. As described by the gyroscope system verification, IAE is expressed as

$$
\mathrm{IAE}=\int_{t_{0}}^{\infty}|e(t)| d t
$$

\section{Simulation Results}

This section presents the results of a numerical simulation performed to evaluate the proposed method. The efficiency of the gyroscope system was evaluated using the sliding mode control method introduced by [1] and the proposed fuzzy sliding mode controller. Performance indices were also used to compare its advantages and disadvantages. In the numerical simulation, the gyroscope system parameters were set to $\beta_{\varphi}=5, I_{1}=1, M_{g}=4, l=0.25, C=0.5$, and $\omega_{2}=2$. Calculations were made at $1 \mathrm{~ms}$ intervals. The initial value of the gyroscope system is set to $x(0)=-0.3, y(0)=-0.1$, and $z(0)=5$. The periodic trajectories designed for the gyroscope system were $\theta_{d}=0.5 \cdot \sin (8 t), \phi_{d}=0.4 \cdot \sin (4 t)$. During the simulation, control signals were added at $t=5 \mathrm{sec}$ to the gyroscope system. According to the sliding mode control in [1], the parameters for sliding surfaces (8) are $\lambda_{1}=\lambda_{2}=3$; the selected parameters for controllers (12) and (13) are $\gamma_{1}=$ $\gamma_{2}=2$. Figures 3 and 4 show the simulation results. The solid line represents the status of the gyroscope system; the dashed line represents the cyclical trace design in Figure 3. Figure 4 is the control signal of the sliding mode of the gyroscope system. The parameters for the proposed fuzzy sliding mode controller sliding surfaces (14) and (15) are set to $k_{1}=2$, $k_{2}=20$, and $k_{3}=10$. The parameters chosen for controller (17) are $\varepsilon_{1}=1.5$, and $\varepsilon_{2}=2, \varepsilon_{s 1}=1, \varepsilon_{s 2}=50$. Figures 5 and 6 show the simulation results. The solid line represents the status of the gyroscope system; the dashed line represents the periodic trajectory in Figure 5. Figure 6 shows the control signal for the fuzzy sliding mode for the gyroscope system. Figure 7 shows the tracking error signal where, the solid line represents the sliding mode control and the dashed line the fuzzy sliding mode control. Table 1 shows the control system performance. Thus, the performance indicators confirm the satisfactory performance of the proposed gyroscope system.

\section{Conclusions}

The study was able to explore the problem from chaotic motion to periodic motion control of the gyroscope system states. The fuzzy sliding mode control was used to drive the system states into the designed states. Introducing the 


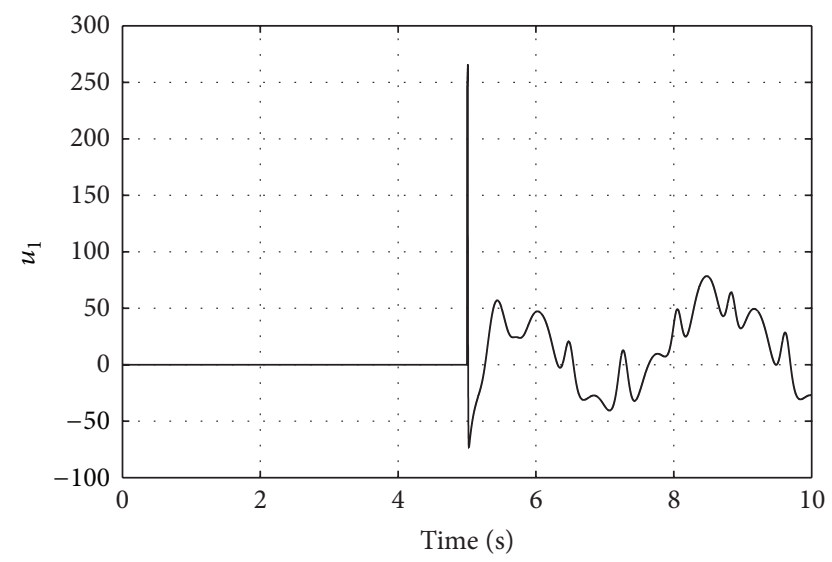

(a)

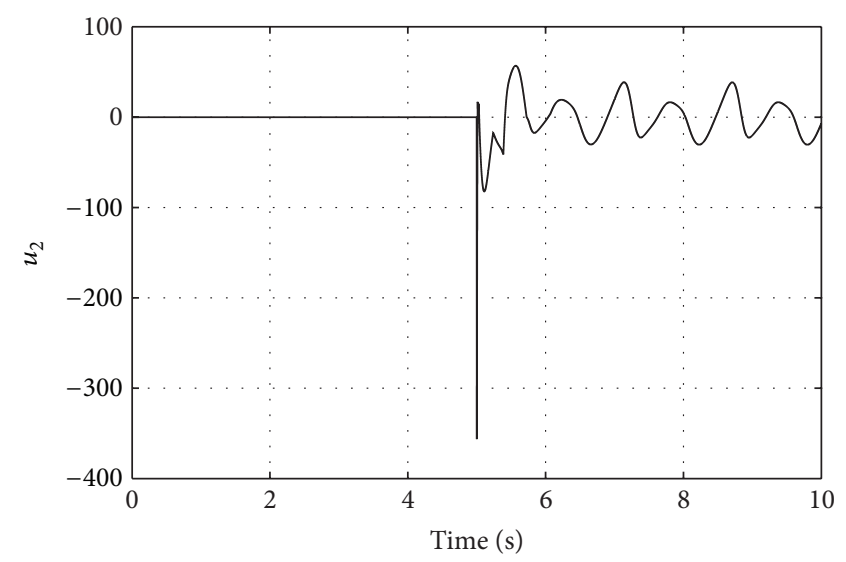

(b)

FIGURE 4: Control input using sliding mode control.

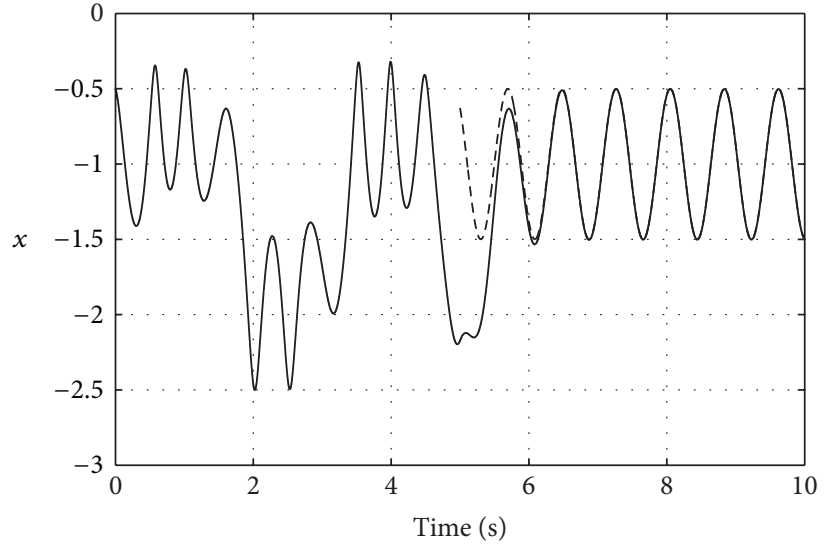

(a)

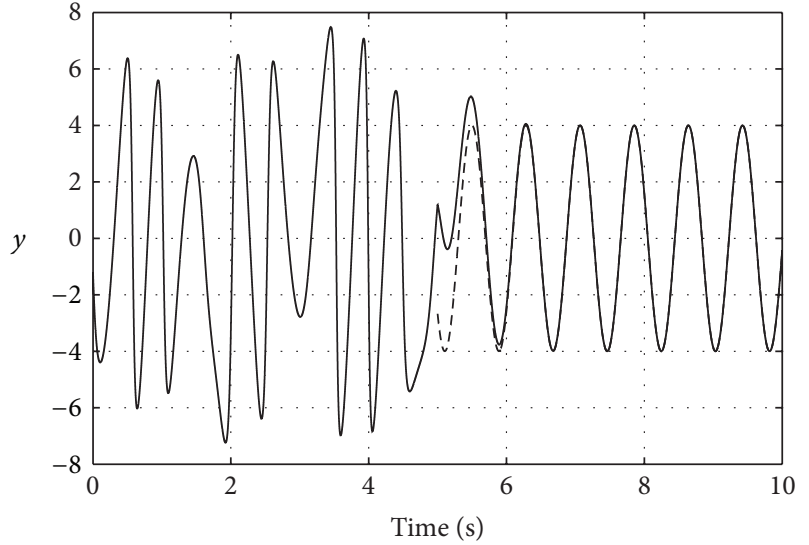

(b)

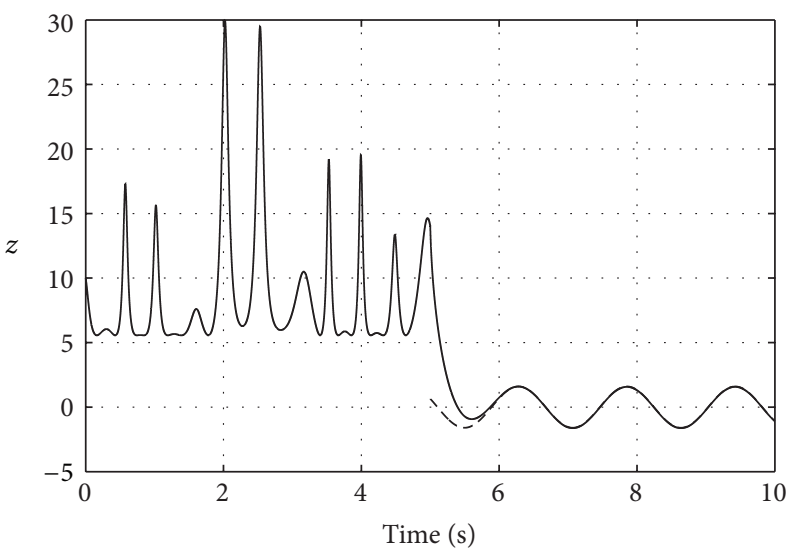

(c)

FIGURE 5: Time response of gyroscope via fuzzy sliding mode control. 


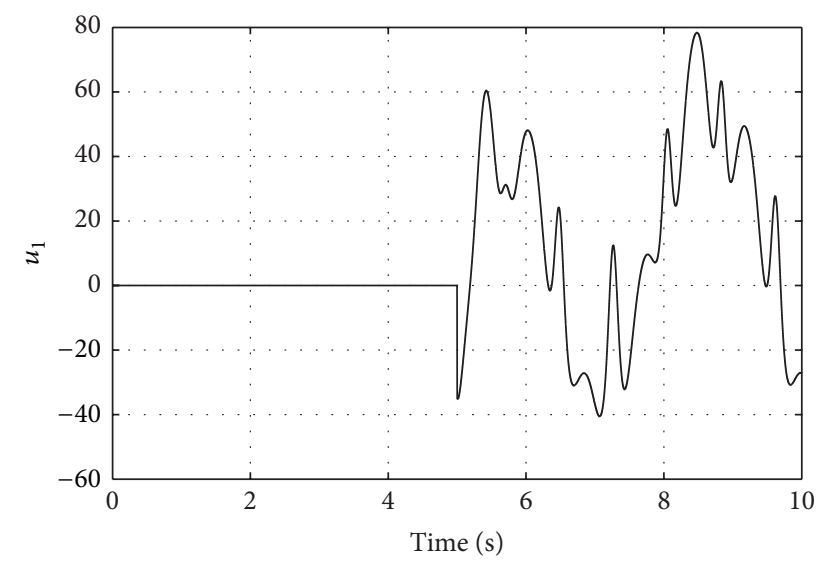

(a)

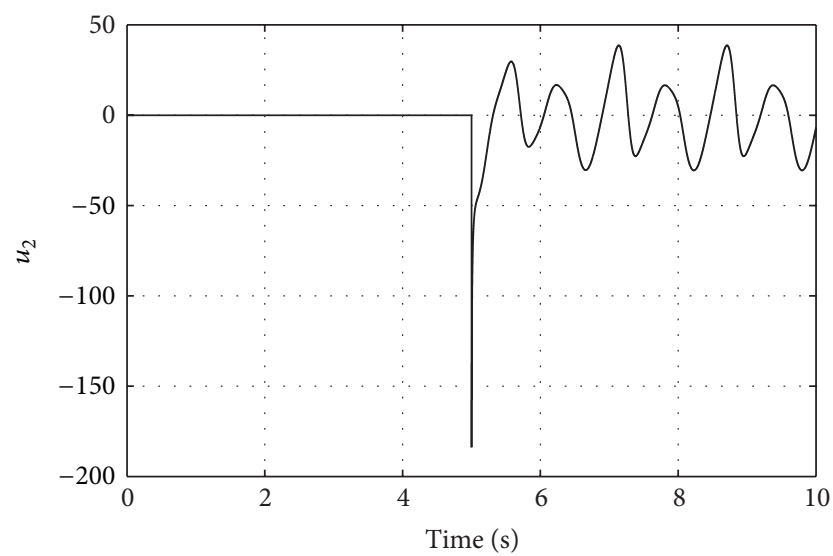

(b)

FIGURE 6: Control input using fuzzy sliding mode control.

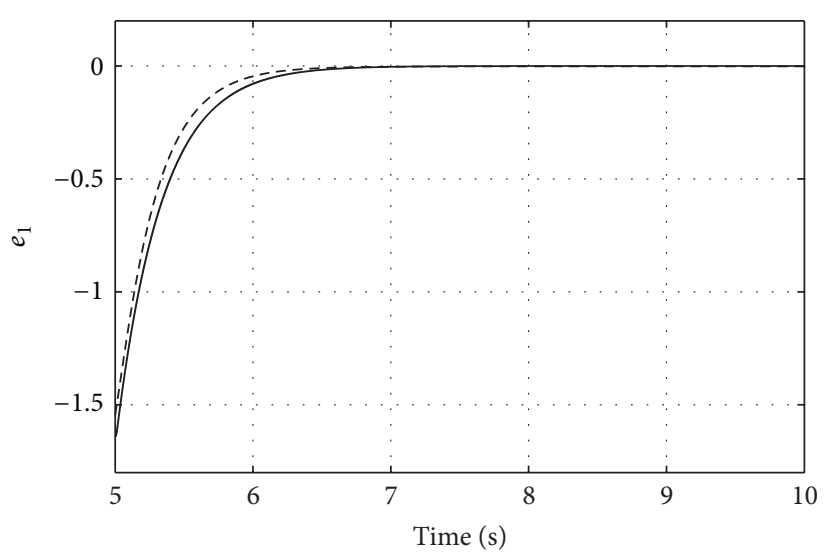

(a)

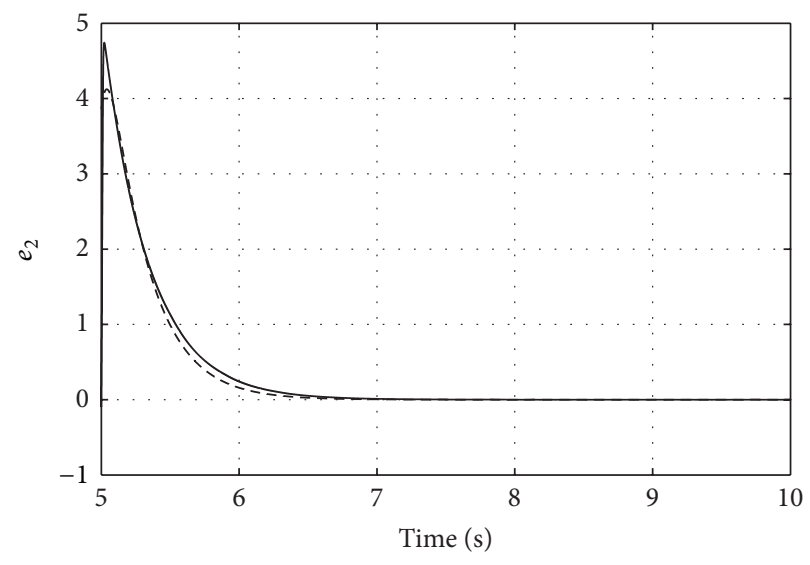

(b)

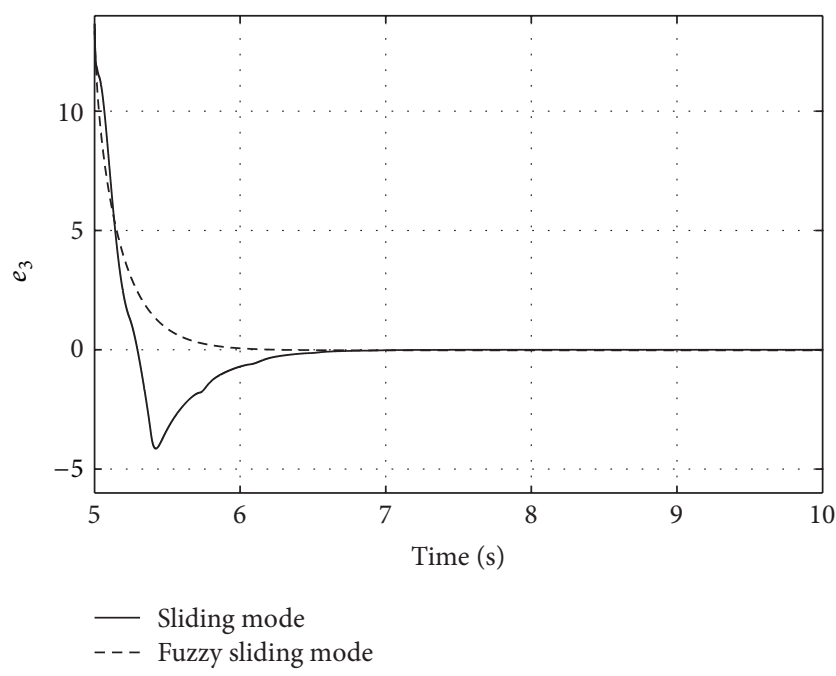

(c)

FiguRE 7: Time response of tracking error states. 
TABLE 1: Performance index of controllers.

\begin{tabular}{lccc}
\hline & IAE of $e_{1}$ & IAE of $e_{2}$ & IAE of $e_{3}$ \\
\hline SMC & 0.5671 & 1.6411 & 1.6413 \\
FSMC & 0.3572 & 1.5711 & 1.3852 \\
\hline
\end{tabular}

Lyapunov stability theory in the design of the fuzzy sliding mode controller enables the use of the proposed control law to ensure a stable and robust gyroscope system. Simulation results show that the proposed fuzzy sliding mode control can solve gyroscope system tracking control problems and does not produce chattering.

\section{Acknowledgment}

This work was supported by the National Science Council of Taiwan, under Contracts NSC 102-2221-E-022-019 and 1012221-E-022-014.

\section{References}

[1] C.-C. Wang and H.-T. Yau, "Nonlinear dynamic analysis and sliding mode control for a gyroscope system," Nonlinear Dynamics, vol. 66, no. 1-2, pp. 53-65, 2011.

[2] Z.-M. Ge and J.-K. Lee, "Chaos synchronization and parameter identification for gyroscope system," Applied Mathematics and Computation, vol. 163, no. 2, pp. 667-682, 2005.

[3] S. Boccaletti, A. Farini, and F. T. Arecchi, "Adaptive synchronization of chaos for secure communication," Physical Review E, vol. 55, no. 5 A, pp. 4979-4981, 1997.

[4] J. Fei, H. Ding, and Y. Yang, "Adaptive sliding mode control of MEMS triaxial gyroscope based on RBF network," in Proceedings of the IEEE International Conference on Mechatronics and Automation (ICMA '11), pp. 331-336, August 2011.

[5] H.-K. Chen, "Chaos and chaos synchronization of a symmetric gyro with linear-plus-cubic damping," Journal of Sound and Vibration, vol. 255, no. 4, pp. 719-740, 2002.

[6] C.-K. Chen, J.-J. Yan, and T.-L. Liao, "Sliding mode control for synchronization of Rössler systems with time delays and its application to secure communication," Physica Scripta, vol. 76, no. 5, pp. 436-441, 2007.

[7] H. T. Yau, T. H. Hung, and C. C. Hsieh, "Bluetooth based chaos synchronization using particle swarm optimization and its applications to image encryption," Sensors, vol. 12, pp. 7468-7484, 2012.

[8] C.-L. Kuo, "Design of a fuzzy sliding-mode synchronization controller for two different chaos systems," Computers \& Mathematics with Applications, vol. 61, no. 8, pp. 2090-2095, 2011.

[9] M. P. Aghababa, S. Khanmohammadi, and G. Alizadeh, "Finitetime synchronization of two different chaotic systems with unknown parameters via sliding mode technique," Applied Mathematical Modelling, vol. 35, no. 6, pp. 3080-3091, 2011.

[10] J. Li and K. D. Kumar, "Decentralized fault-tolerant control for satellite attitude synchronization," IEEE Transactions on Fuzzy Systems, vol. 20, no. 3, pp. 572-586, 2012.

[11] H.-T. Yau, "Chaos synchronization of two uncertain chaotic nonlinear gyros using fuzzy sliding mode control," Mechanical Systems and Signal Processing, vol. 22, no. 2, pp. 408-418, 2008.
[12] D. I. R. Almeida, J. Alvarez, and J. G. Barajas, "Robust synchronization of Sprott circuits using sliding mode control," Chaos, Solitons and Fractals, vol. 30, no. 1, pp. 11-18, 2006.

[13] C.-L. Kuo, C.-C. Wang, and N.-S. Pai, "Design of variable structure synchronization controller for two different hyperchaotic systems containing nonlinear inputs," Journal of Applied Sciences, vol. 9, no. 14, pp. 2635-2639, 2009. 


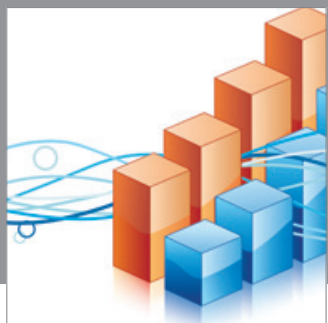

Advances in

Operations Research

mansans

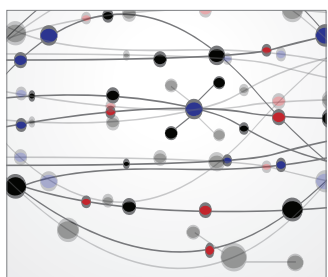

The Scientific World Journal
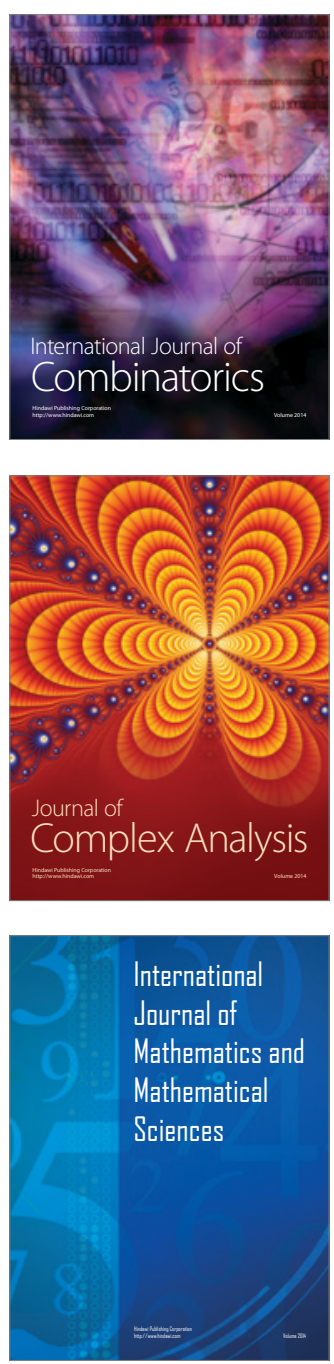
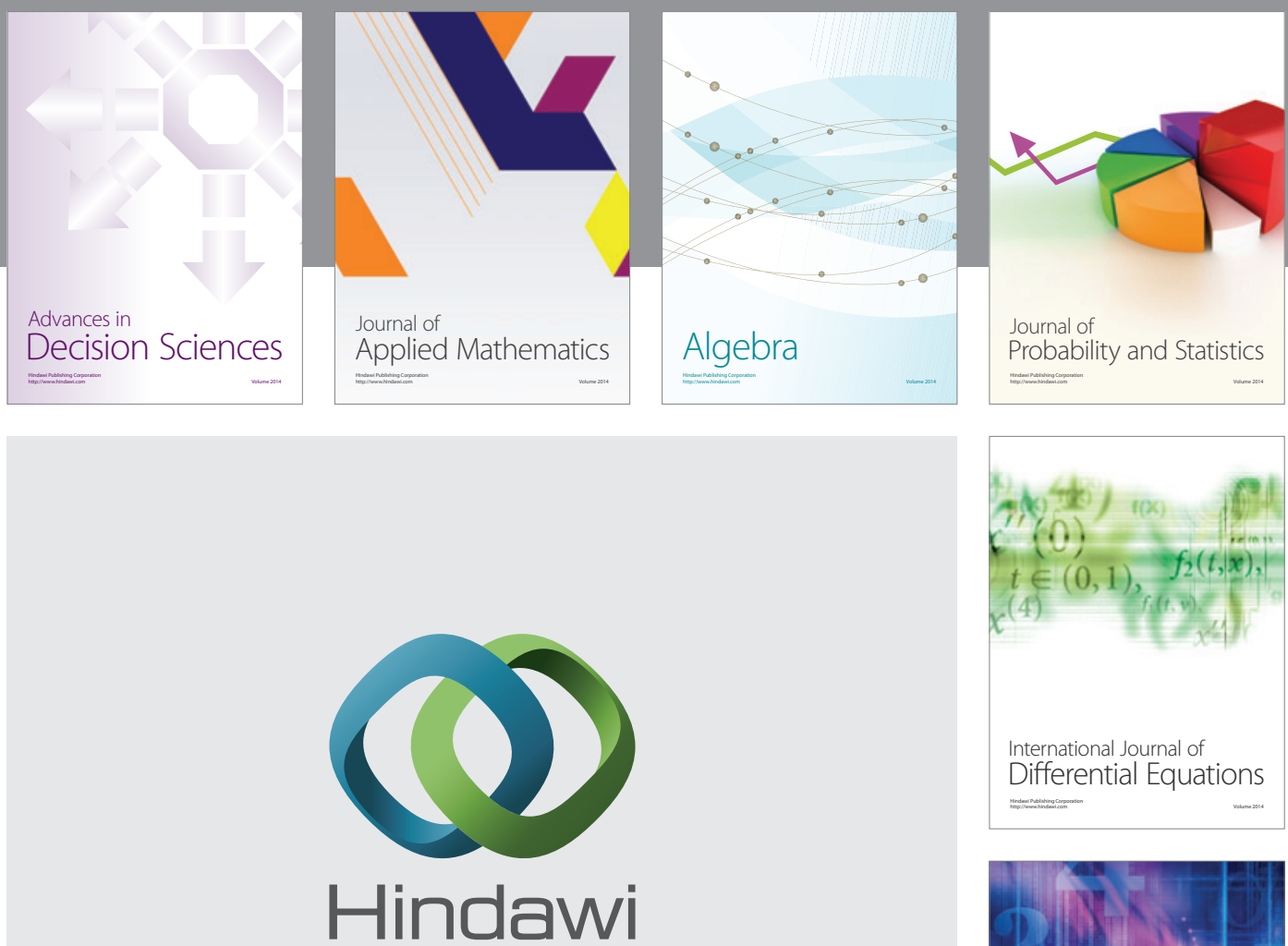

Submit your manuscripts at http://www.hindawi.com
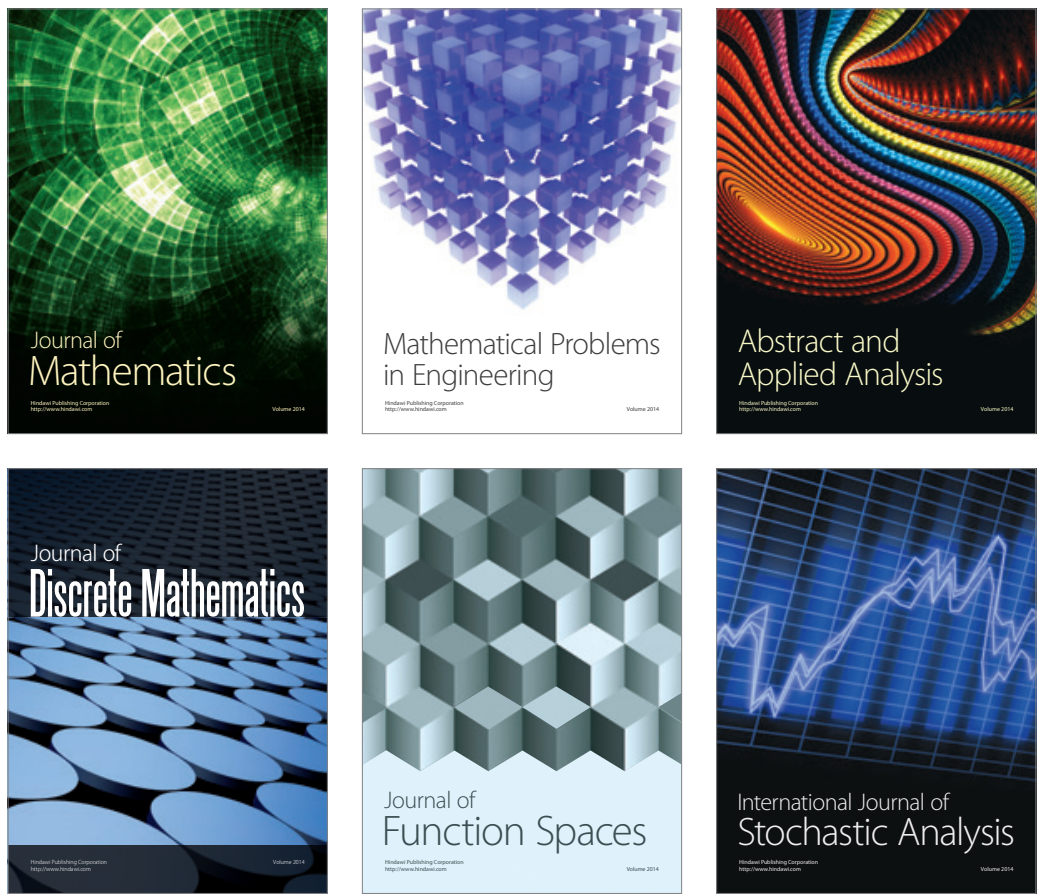

Journal of

Function Spaces

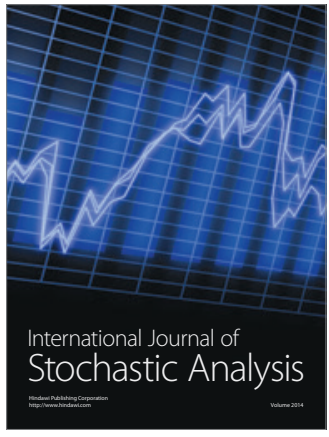

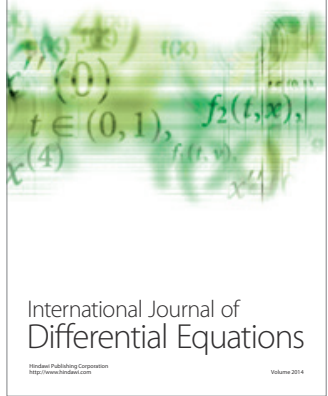
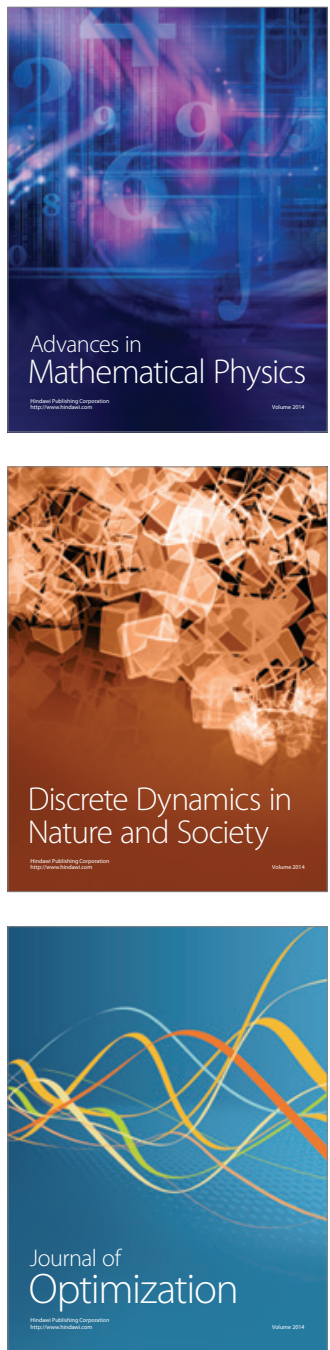